\title{
QTLS ASOCIADOS CON LA TOLERANCIA A SEQUÍA EN UNA POBLACIÓN DE MAÍZ TROPICAL UTILIZANDO LÍNEAS Y CRUZAS DE PRUEBA
}

\author{
QTL ASSOCIATED WITH DROUGHT TOLERANCE IN A POPULATION \\ OF TROPICAL MAIZE USING LINES AND TEST CROSSES
}

\author{
Victor Vazquez-Pozos, Eliud Serrano-Flores, Serafín Cruz-Izquierdo* y Ricardo Lobato-Ortiz
}

\begin{abstract}
Colegio de Postgraduados, Campus Montecillo, Postgrado en Recursos Genéticos y Productividad-Genética, Montecillo, Texcoco, Estado de México, México.

*Autor de correspondencia (sercruz@colpos.mx)
\end{abstract}

\section{Resumen}

Para avanzar en el mejoramiento para tolerancia al estrés hídrico en maíz (Zea mays L.) es necesario acumular información de loci de caracteres cuantitativos (QTLs) asociados con este carácter en líneas elite adaptadas a condiciones de sequía que puedan emplearse para los escenarios de cambio climático. En este estudio se evaluaron en los ambientes de sequía y riego un grupo de líneas dobles haploides derivadas de la cruza LPSC7F64 × CML495 y un grupo de cruzas de prueba hacia la línea CML494. Los resultados indicaron que en las dos condiciones de humedad, el índice de vegetación diferencial normalizado (NDVI) fue el parámetro más asociado con el rendimiento $\left(r_{q}>\right.$ $0.85)$, al igual que el índice que combina el vigor temprano, vigor final y la senescencia (COMBO; $r_{q}>0.68$ ). Se identificaron QTLs para vigor temprano bajo condiciones de sequía (Bin 1.03) que explican el $10.9 \%$ de la variación fenotípica (\% VF) (aditividad + 26), al igual que COMBO bajo sequía (Bin 1.02/1.03) con el $7.49 \%$ VF (aditividad + 28). Así mismo, se ubicaron varios QTLs para sequía y riego (Bin 4.05), donde sobresalieron COMBO_LR (13\% VF) y área bajo la curva de la senescencia (AUCSEN) (13.81\% VF); además, se identificaron otros QTLs de COMBO_LR en la Bin 5.05 (11.6\% VF) y COMBO_LR en la Bin 7.03 (9.9\% VF). Adicionalmente, se localizaron QTLs para rendimiento (aditividad $0.12 \mathrm{t} \mathrm{ha}^{-1}$ ). Estos QTLs pueden ser útiles para mejorar el rendimiento del maíz bajo condiciones de sequía y riego.

Palabras clave: Zea mays, NDVI, QTL, sequía, trópico.

\section{SUMMARY}

In order to make progress in improving tolerance to water stress in maize (Zea mays L.) it is necessary to accumulate information on quantitative trait loci (QTL) associated with this character in elite inbred lines adapted to drought conditions that can be used for scenarios of climate change. In this study, a group of doubled haploid inbred lines derived from the LPSC7F64 $\times$ CML495 cross and a group of test crosses by the CML494 inbred line were evaluated under dry and irrigated environments. Results indicated that under both moisture conditions the normalized differential vegetation index (NDVI) was the parameter most associated with yield $\left(r_{g}>0.85\right)$, as well as the index that combines early vigor, final vigor and senescence (COMBO; $\left.r_{g}>0.68\right)$. QTL were identified for early vigor under drought conditions (Bin 1.03), explaining 10.9 $\%$ of the phenotypic variation (\% PV) (additivity +26 ), as well as COMBO under drought (Bin 1.02/1.03) with 7.49 \% PV (additivity + 28). Likewise, several QTL were also identified for drought and irrigation (Bin 4.05), where COMBO_LR $(13 \% \mathrm{PV})$ and area under the senescence curve (AUCSEN_DS) (13.8\% PV) were remarkable. Furthermore, other COMBO_LR QTL were located at Bin 5.05 (11.6 \% PV) and COMBO_LR at Bin 7.03 (9.9\% PV). Additionally, QTL were found for yield (additivity $0.12 \mathrm{t} \mathrm{ha}^{-1}$ ). These QTL can be useful for improving maize yield under both drought and irrigation conditions.

Index words: Zea mays, drought, NDVI, QTL, tropic.

\section{INTRODUCCIÓN}

Los cereales, como el maíz (Zea mays L.), son el principal alimento y fuente de energía para los habitantes de la mayoría de los países en desarrollo. Cerca del $60 \%$ de las calorías se derivan directamente de ellos y en los países más pobres se llega hasta el 80 \% (Awika, 2011). Entre los tres principales cereales (maíz, trigo y arroz), el maíz contribuye con más del $40 \%$ de la producción mundial con 1,038 millones de toneladas (FAOSTAT, 2015); además, por su alto potencial de rendimiento, es reconocido como un cultivo que puede contribuir en alto grado a la seguridad alimentaria (Almeida et al., 2014).

Se ha comprobado que el cambio climático se está presentando con un aumento en los fenómenos meteorológicos extremos como la temperatura y la variabilidad de las Iluvias (Olivera, 2013). En el informe del Panel Intergubernamental del Cambio Climático se menciona que las sequías se intensificarán durante este siglo en algunas zonas de Europa Central, América Central, México, Noreste de Brasil y Sur de África debido a la reducción de las precipitaciones y al aumento de la evapotranspiración (IPCC, 2012). La escasez de agua es el factor más limitante del medio ambiente para la productividad del maíz (Inzunza-Ibarra et al., 2018) en las regiones tropicales y subtropicales (Messmer et al., 2011). Se estima que el rendimiento se reduce hasta en $39 \%$ cuando hay una escasez de agua de $40 \%$ aproximadamente (Daryanto et al., 2016).

En la historia del mejoramiento por hibridación se ha demostrado que la tolerancia al estrés está más asociada 
a la ganancia genética que a la heterosis en los genotipos más recientes (Duvick, 2005; Reyes et al., 2015) debido a que se ha mejorado la eficiencia del uso del agua y el índice de cosecha. El mejoramiento genético por selección para la tolerancia a la sequía mediante rendimiento de grano es ineficiente debido a la baja heredabilidad de ambos caracteres, por lo que se sugiere el uso de variables secundarias, que proporcionan información de la forma en que el rendimiento cambia en condiciones de sequía. Idealmente, un carácter secundario debe de estar correlacionado con el rendimiento de grano en condiciones de sequía, con variación genética y altamente heredable; también debe ser fácil, barato y rápido de observar o medir de forma no destructiva, estable durante el periodo de medición y no asociado con la pérdida de rendimiento en condiciones sin estrés (Lafitte et al., 2003).

Debido a que la tolerancia a la sequía es de herencia cuantitativa, controlada por muchos genes, resulta apropiado apoyarse en técnicas moleculares para identificar los principales procesos fisiológicos asociados con la mejora del rendimiento y la determinación de las asociaciones gen-fenotipo que permitan aumentar la eficiencia de la selección. El mejoramiento asistido por marcadores moleculares es una herramienta que agiliza el avance genético (Ribaut y Ragot, 2007), apoyado por loci de carácter cuantitativo. Los QTLs permite conocer la importancia de una región del genoma asociada a un carácter. En maíz se han descrito varios QTLs asociados a condiciones de estrés hídrico, como intervalo de floración, enrollamiento de la hoja, senescencia, vigor, entre otros (Almeida et al., 2014; Lu et al., 2012; Messmer et al., 2011; Trachsel et al., 2016).

La detección de QTLs en grupos de líneas puede ser no del todo buena debido a la carga genética para el caso del maíz, por lo que una solución es la evaluación de éstas en combinación con un probador (Frascaroli et al., 2009; Ramírez Díaz et al., 2019) y así detectar QTLs consistentes. El objetivo de este trabajo fue identificar QTLs para la tolerancia a sequía en una población de maíz de trópico. La consecución de dicho objetivo incluyó i) estimación de la heredabilidad y la relación de los rasgos secundarios con el rendimiento de grano bajo ambientes de sequía y riego, ii) identificación de las regiones genómicas que influyen en los caracteres secundarios de los regímenes hídricos, y iii) detección de regiones del genoma con QTLs clave para posteriores trabajos de introgresión asistida por marcadores de tolerancia a la sequía en maíz tropical.

\section{MATERIALES Y MÉTODOS}

\section{Material vegetal}

El material utilizado fue un grupo de 166 líneas de maíz biparentales obtenidas por la técnica de dobles haploides a partir de las líneas LPSC7F64 (La Posta Sequía C7 F64-26-2-2-B-B-B) y CML495, además de 196 cruzas de prueba obtenidas utilizando la línea CML494. La LPSC7F64 es una línea que fue derivada de la población La Posta Sequía, de grano blanco dentado del patrón heterótico B; es un sintético de la raza Tuxpeño, adaptado a la condición del trópico bajo, desarrollado mediante siete ciclos de selección recurrente bajo condiciones de sequía. La línea CML495 es de grano blanco cristalino desarrollada para condiciones de trópicos bajos del patrón heterótico A. El probador CML494 es una línea de grano blanco dentado obtenido de la población La Posta Sequía Ciclo 4 Familia 7 , de grupo heterótico AB (http://apps.cimmyt.org).

\section{Sitios experimentales}

El experimento se estableció en la estación experimental del Centro International de Mejoramiento de Maíz y Trigo (CIMMYT) en Tlaltizapán, Morelos (18 41' N, $99^{\circ} 08^{\prime}$ O, 940 msnm) e Instituto Nacional de Investigaciones Forestales, Agrícolas y Pecuarias (INIFAP) de Iguala, Guerrero ( $18^{\circ} 20^{\prime}$ $\mathrm{N}, 99^{\circ} 30^{\prime}$ O, $732 \mathrm{msnm}$ ). La siembra se realizó durante la temporada de invierno seco 2013 y 2014 cuando normalmente no hay lluvias y se depende de la irrigación.

\section{Diseño y unidad experimental}

Se utilizó un diseño experimental a-látice $(0,1)$ con bloques incompletos y dos repeticiones. La unidad experimental fue de un surco de $4.5 \mathrm{~m}$ y $0.75 \mathrm{~m}$ entre surcos, un espaciamiento entre plantas de $0.2 \mathrm{~m}$, para una densidad de 66.6 plantas $\mathrm{m}^{-2}$.

\section{Manejo experimental}

Se realizaron prácticas culturales convencionales en las parcelas de ensayo y los riegos fueron mediante goteo. El estrés por sequía se indujo de acuerdo con el protocolo de CIMMYT (Bänziger et al., 2012) a los 12-15 días antes de la antesis ( 190 UC). Para el caso de un estrés hídrico severo indicado por un intervalo de floración (IF) mayor de $5 \mathrm{~d}$ en promedio del ensayo, el riego se aplicó $7 \mathrm{~d}$ después de finalizada la antesis, mientras que un segundo riego se aplicó tres semanas después de la finalización de la antesis. 
En el caso de estrés hídrico moderado, se aplicó un solo riego durante el periodo de llenado del grano dos semanas después de la finalización de la antesis. El monitoreo de la humedad se realizó tres veces por semana con una sonda de humedad del suelo PR2 / 6 (Delta-T Devices, Cambridge, Reino Unido) a profundidades de 10, 20, 30, 40, 60 y 100 $\mathrm{cm}$ para la programación de riegos en el tratamiento de estrés por sequía.

\section{Datos fenotípicos}

Las variables fenotípicas se obtuvieron a lo largo del ciclo del cultivo. El índice de vegetación de diferencia normalizada (NDVI) se capturó a las dos, cuatro, cinco y seis semanas después de la siembra con un RT-Greenseeker (Trimble, Ukia, CA, EUA). El NDVI fue medido en cada unidad experimental a una altura de $0.8 \mathrm{~m}$ caminando con el sensor en medio de los surcos. El NDVI fue calculado de la siguiente manera:

$$
N D V I=\frac{I R C-R}{I R C+R}
$$

donde: IRC es la reflectividad en el infrarrojo cercano y $R$ es la reflectividad en el rojo.

Los días a antesis (DA) se obtuvieron con el número de días desde la siembra hasta el $50 \%$ de las plantas con dehiscencia de polen. El intervalo de floración fue medido por el intervalo de tiempo en días entre la floración masculina y la femenina. La altura de planta (AP) se midió en $\mathrm{cm}$ al momento de la floración desde el suelo hasta la última hoja. La senescencia se registró a las dos, cuatro y seis semanas después de la floración mediante una calificación visual con una escala de 1 (sin senescencia) a 9 (senescencia completa). Para NDVI y senescencia, el área bajo la curva (AUC) se calculó mediante la integración de una función polinomial de segundo grado ajustada a mediciones individuales tomadas antes (por NDVI) o después de la floración (por senescencia). Se adicionó un indice que integra el vigor temprano (NDVI), vigor final (altura de planta) y senescencia, denominado COMBO, que se calculó de la siguiente manera:

$$
\text { COMBO }=\frac{A P \times A U C N D V I}{A U C S E N}
$$

donde: AP es la altura de planta a floración, AUCNDVI es el área bajo la curva de NDVI y AUCSEN es el área bajo la curva de la senescencia.

El rendimiento de grano se calculó con el peso de grano ajustado a una humedad de $12.5 \%$.

\section{Análisis estadístico}

Los componentes de varianza fueron estimados con máxima verosimilitud lineal restringida mediante META- $R$ 2.5 (Alvarado et al., 2015). El modelo estándar lineal mixto para la variable respuesta estuvo representado por:

$$
Y=\mu+G_{i}+E_{j}+G E_{i j}+R(E)_{k j}+B I(R E)_{b k j}+e_{i j k b}
$$

En el que $\mu$ es la media general, $G_{i}$ es el efecto del i-ésimo genotipo ( $i=166$ y 196 líneas y cruzas de prueba), $E_{j}$ es el efecto del $j$-ésimo ambiente (CP_R: $j=2$, CP_S: $j=4$, Líneas DH_R $j=2$ y Líneas DH_S $j=2), G E_{i j}$ es el efecto de interacción del $i$-ésimo genotipo con el $j$-ésimo ambiente, $R(E)_{k j}$ es el efecto de la k-ésima repetición dentro del j-ésimo ambiente, $B I(R E)_{b k j}$ es el efecto de los bloques incompletos dentro de la k-ésima repetición en el j-ésimo ambiente. El efecto ambiente no fue considerado aleatorio. Los mejores predictores lineales insesgados (BLUP) se calcularon para las líneas y cruzas de prueba en las dos condiciones de riego por separado.

La heredabilidad en sentido amplio fue estimada como:

$$
H^{2}=\sigma_{g}^{2} /\left(\sigma_{g}^{2}+\frac{\sigma_{q \times e}^{2}}{e}+\frac{\sigma_{e}^{2}}{r}\right)
$$

donde: $\sigma_{g}^{2}$ es la varianza genotípica, $\sigma_{g \times e}^{2}$ es la varianza de la interacción genotipo $\times$ ambiente, $\sigma^{2}{ }_{e}$ es la varianza residual, e es el número de ambientes y $r$ es el número de repeticiones por ambiente.

La correlación genética $\left(r_{q}\right)$ entre las características se calculó con la siguiente ecuación:

$$
r_{g}=\sigma_{g\left(i^{\prime}\right)} / \sigma_{g(i)} \sigma_{g(i)}
$$

donde: $\sigma_{g\left(i^{i}\right)}$ es la covarianza genotípica entre cada par de caracteres y $\sigma_{g(i)} \sigma_{g(i)}$ es el producto de las desviaciones estándar genotípicas de cada carácter.

Las correlaciones fenotípicas entre las características se calcularon con base en los BLUP como los coeficientes de correlación de Pearson.

\section{Genotipificación y construcción de mapa de ligamiento}

Se tomaron 12 muestras de hoja por cada una de las líneas usadas en el estudio. Para el aislamiento del ADN se usó el procedimiento de CTAB (CIMMYT, 2005). El ADN de todas las muestras se envió a Cornell University Biotechnology Resource Center (Ithaca, NY, EUA) para genotipeo por secuenciación (GBS), donde se aplicó el protocolo de Elshire et al. (2011). El ADN genómico se 
digirió con la enzima de restricción ApaK1. Las bibliotecas de GBS fueron construidas en 96-plex y secuenciadas en un equipo Illumina HiSeq 2000 (Illumina, Inc., San Diego, California, EUA) (Glaubitz et al., 2014; Zhang et al., 2015). Los marcadores de polimorfismo de nucleótido individual (SNP) se filtraron e imputaron en el programa TASSEL GBS y se usó como referencia el genoma de la línea B73 (Glaubitz et al., 2014). El mapa de ligamiento se construyó en ICIMv4 con 476 SNP; para estimar la distancia del mapa se usó la función de Kosambi (Kosambi, 1943).

\section{Análisis de QTLs}

El mapeo de intervalo compuesto fue realizado con ICIMv4 (Li et al., 2007). Se incluyeron 174 genotipos en el mapa con una convergencia de $1 \mathrm{cM}$. Un QTL con valor LOD mayor o igual que 2.5 fue considerado como significativo $(P \leq 0.05)$. Un QTL para diferentes caracteres fue declarado común cuando sus posiciones con altas puntuaciones LOD (pico) se ubicaban en la misma posición. El efecto aditivo se obtuvo con ICIMv4. El signo de efectos aditivos fue usado para identificar el origen del alelo favorable. Aditividad positiva indica un efecto de sustitución proveniente del progenitor recurrente (CML495) y aditividad negativa indica el efecto de sustitución proviene del donador (LPSC7F64). $\mathrm{R}^{2}$ representa el porcentaje de la varianza fenotípica explicada por todos los QTLs detectados para cada carácter (Zeng et al., 1999).

\section{RESULTADOS Y DISCUSIÓN}

Las estimaciones de medias, componentes de varianza y heredabilidad de las líneas DH se muestran en el Cuadro 1 y de las cruzas de prueba en el Cuadro 2 . El rendimiento promedio de la población de líneas $\mathrm{DH}$ bajo riego y sequia fue de 1.9 y $0.7 \mathrm{tha}^{-1}$, mientras que en las cruzas de prueba fue de 4.11 y 3.11 tha $^{-1}$ para riego y sequía, respectivamente. La heredabilidad fue de 0.40 y 0.56 para líneas bajo riego y sequía, respectivamente (Figura 1), mientras que en cruzas de prueba (CP) ésta fue de 0.61 y 0.28 para riego y sequía, respectivamente. La reducción del rendimiento por efecto de la sequía fue de $63.16 \%$ para las líneas DH y de $24.33 \%$ para las cruzas de prueba.

En las CP la antesis fue más temprana que en las líneas DH. Las heredabilidades fueron moderadas y altas $\left(H^{2}>\right.$ 0.56) y los coeficientes de variación bajos (< $2.5 \%)$. La antesis se presentó antes en las cruzas de prueba bajo sequía que en las de riego (Figura 1), con lo que se presenta un escape a la sequía de aproximadamente $4 \mathrm{~d}$.

El intervalo de floración presentó diferencias entre genotipos únicamente en líneas $\mathrm{DH}$ bajo sequía, con un intervalo de -8 a 2.3 d mientras que en las CP en sequía el intervalo fue reducido, de 1.3 a 2.3 d. Las líneas bajo sequía presentaron una heredabilidad intermedia $\left(H^{2}=\right.$ 0.68), mientras que las cruzas de prueba presentaron baja heredabilidad $\left(H^{2}=0.21\right)$.

La altura de planta presentó diferencias significativas entre las poblaciones en las dos condiciones, donde las poblaciones bajo sequía presentaron valores mayores que las de riego para las CP. La diferencia en altura promedio de la población de líneas DH y CP bajo riego $\left(H^{2}=0.69\right.$ y 0.37$)$ y sequía $\left(H^{2}=0.74\right.$ y 0.84$)$ fue de 10.8 y $29.8 \mathrm{~cm}$, respectivamente.

La calificación de SEN1 no presentó diferencias entre genotipos para la población CP, y las heredabilidades fueron bajas $\left(H^{2}<0.24\right)$. Mientras que en las calificaciones de SEN2 sólo en líneas $\mathrm{DH}$ bajo riego y sequía se presentaron diferencias con medias de calificación de 2.8 para riego $\left(H^{2}\right.$ $=0.34)$ y sequía de $4.8\left(H^{2}=0.42\right)$. En SEN3 se presentaron diferencias entre genotipos en las diferentes poblaciones de riego y sequía con heredabilidades intermedias, en líneas DH la media bajo riego fue de $5.5\left(H^{2}=0.51\right)$ y para sequia de $7.8\left(H^{2}=0.46\right)$, en tanto que en cruzas de prueba en riego fue de $3.2\left(H^{2}=0.37\right)$ y en sequía $7.3\left(H^{2}=0.40\right)$. Los resultados en AUCSEN revelaron diferencias entre genotipos en las líneas $\mathrm{DH}$ bajo riego $\left(H^{2}=0.48\right)$ y sequía $\left(H^{2}=0.51\right)$, mientras que las CP bajo sequía no presentaron diferencias entre genotipos $\left(H^{2}=0.28\right)$.

Los valores de NDVI en las CP bajo riego fueron mayores, debido quizá a una mejor fertilidad del suelo. Las heredabilidades van de forma creciente de NDVI1 a NDVI4 $\left(H^{2}=0.29\right.$ a 0.74). AUCNDVI presenta heredabilidad alta $\left(H^{2}\right.$ $=0.63$ a 0.75 ).

Los resultados de COMBO sólo se presentan para líneas DH bajo riego $\left(H^{2}=0.60\right)$ y cruzas de prueba en sequía $\left(H^{2}\right.$ $=0.65)$, donde se obtuvieron diferencias entre genotipos.

\section{Correlaciones de las características secundarias con el rendimiento dentro de poblaciones y condiciones de riego}

En el Cuadro 3 se presentan las correlaciones de las características secundarias con el rendimiento. En las líneas $\mathrm{DH}$ bajo riego la mayoría de las características tuvieron asociaciones altas con el rendimiento, con excepción del intervalo de floración y senescencia. Para las CP y DH en riego todas las calificaciones de senescencia, AUCSEN, NDVI (todas las capturas), AUCNDVI y COMBO presentaron correlaciones altas y positivas; IF presentó correlación intermedia, mientras que el resto no presentó correlación. En las Líneas DH en sequía la altura de planta presentó la mayor asociación con el rendimiento con una correlación 
Cuadro 1. Estimación de medias, componentes de la varianza y heredabilidad del rendimiento y caracteres secundarios de líneas $\mathrm{DH}$ bajo riego $(\mathrm{R})$ y sequía $(\mathrm{S})$.

\begin{tabular}{|c|c|c|c|c|c|c|c|}
\hline Característica & Condición & Media & $\sigma_{g}^{2}$ & $\sigma_{g \times e}^{2}$ & $\sigma_{\mathrm{e}}^{2}$ & $H^{2}$ & CV (\%) \\
\hline \multirow{2}{*}{$\mathrm{RG}\left(\mathrm{tha} \mathrm{h}^{-1}\right)$} & $\mathrm{R}$ & 1.9 & 0.15 & $0.29 * *$ & 0.29 & 0.40 & 28.1 \\
\hline & $S$ & 0.7 & 0.04 & $0.02 *$ & 0.09 & 0.56 & 41.8 \\
\hline \multirow{2}{*}{$\mathrm{DA}(\mathrm{d})$} & $\mathrm{R}$ & - & - & - & - & - & - \\
\hline & $S$ & 89.1 & 8.96 & $1.57 * *$ & 4.76 & 0.82 & 2.45 \\
\hline \multirow{2}{*}{ IF (d) } & $\mathrm{R}$ & 0.28 & $3.6 \times 10^{-13}$ & $3.85 * *$ & 13.05 & $7.0 \times 10^{-14}$ & 1292 \\
\hline & $S$ & -1.1 & 2.91 & $0.58 *$ & 4.37 & 0.68 & 199.9 \\
\hline \multirow{2}{*}{$\mathrm{AP}(\mathrm{cm})$} & $\mathrm{R}$ & 111.3 & 108.63 & 1.90 & 146.35 & 0.74 & 10.9 \\
\hline & $S$ & 100.5 & 69.36 & 0.00 & 124.48 & 0.69 & 11.1 \\
\hline \multirow{2}{*}{ SEN1 (1-10) } & $\mathrm{R}$ & 1.4 & 0.02 & $0.03 * *$ & 0.21 & 0.24 & 31.9 \\
\hline & S & 2.4 & 0.03 & $0.06 *$ & 0.33 & 0.23 & 23.7 \\
\hline \multirow{2}{*}{ SEN2 (1-10) } & $\mathrm{R}$ & 2.8 & 0.04 & $1.6 \times 10^{-3}$ & 0.31 & 0.34 & 19.9 \\
\hline & S & 4.8 & 0.06 & $0.05 \star \star$ & 0.25 & 0.42 & 10.4 \\
\hline \multirow{2}{*}{ SEN3 (1-10) } & $\mathrm{R}$ & 5.5 & 0.31 & $0.26 * *$ & 0.66 & 0.51 & 15 \\
\hline & S & 7.8 & 0.35 & $0.20 *$ & 1.27 & 0.46 & 14.5 \\
\hline \multirow{2}{*}{ AUCSEN } & $\mathrm{R}$ & 91.5 & 58.18 & $37.36 * *$ & 173.35 & 0.48 & 14.4 \\
\hline & $S$ & 142.1 & 85.82 & $43.01 *$ & 239.70 & 0.51 & 10.9 \\
\hline \multirow{2}{*}{ NDVI1 } & $\mathrm{R}$ & 0.2 & $2.1 \times 10^{-4}$ & $2.0 \times 10^{-4 * *}$ & $5.1 \times 10^{-4}$ & 0.49 & 9.64 \\
\hline & S & - & - & - & - & - & \\
\hline \multirow{2}{*}{ NDVI2 } & $\mathrm{R}$ & 0.4 & $8.7 \times 10^{-4}$ & $4.0 \times 10^{-4 \star}$ & $1.9 \times 10^{-3}$ & 0.56 & 10.72 \\
\hline & S & - & - & - & - & - & \\
\hline \multirow{2}{*}{ NDVI3 } & $\mathrm{R}$ & 0.4 & $1.4 \times 10^{-3}$ & $3.7 \times 10^{-4 \star}$ & $3.2 \times 10^{-3}$ & 0.60 & 13.69 \\
\hline & S & - & - & - & - & - & \\
\hline \multirow{2}{*}{ NDVI4 } & $\mathrm{R}$ & 0.6 & $1.4 \times 10^{-3}$ & $2.6 \times 10^{-4 \star}$ & $2.7 \times 10^{-3}$ & 0.63 & 9.09 \\
\hline & $S$ & - & - & - & - & - & \\
\hline \multirow{2}{*}{ AUCNDVI } & $\mathrm{R}$ & 11.3 & 0.72 & $0.21 *$ & 1.25 & 0.63 & 9.85 \\
\hline & S & - & - & - & - & - & \\
\hline \multirow{2}{*}{ СОМВО } & $\mathrm{R}$ & 15.2 & 7.24 & $4.91 * *$ & 9.68 & 0.60 & 20.43 \\
\hline & S & - & - & - & - & - & \\
\hline
\end{tabular}

RG: rendimiento de grano, DA: días a antesis, IF: intervalo de floración, AP: altura de planta, SEN: senescencia, NDVI: índice de vegetación de diferencia normalizada, AUC (AUCSEN y AUCNDVI): área bajo la curva, $\sigma^{2}$ : varianza genética, $\sigma^{2}$ y e : varianza genotipo $\times$ ambiente, $\sigma^{2}$ : varianza residual, $\mathrm{H}^{2}$ : heredabilidad en sentido amplio, $\mathrm{CV}$ : coeficiente de variación. -; información no disponible, líneas que no prosperaron en una localidad se eliminaron del análisis combinado. *: significancia $\mathrm{P} \leq 0.05$, **: significancia $\mathrm{P} \leq 0.01$ 
Cuadro 2. Estimación de medias, componentes de la varianza y heredabilidad del rendimiento y caracteres secundarios de cruzas de prueba bajo riego (R) y sequía (S).

\begin{tabular}{|c|c|c|c|c|c|c|c|}
\hline Característica & Condición & Media & $\sigma_{g}^{2}$ & $\sigma_{g \times e}^{2}$ & $\sigma_{\mathrm{e}}^{2}$ & $\mathrm{H}^{2}$ & $\mathrm{CV}(\%)$ \\
\hline \multirow{2}{*}{$R G\left(\mathrm{t} \mathrm{ha}^{-1}\right)$} & $\mathrm{R}$ & 4.11 & 0.32 & 0 & 0.82 & 0.61 & 22.08 \\
\hline & $S$ & 3.11 & 0.05 & $0.15 * *$ & 0.68 & 0.28 & 26.5 \\
\hline \multirow{2}{*}{ DA (d) } & $\mathrm{R}$ & 84.8 & 1.45 & 0.37 & 3.82 & 0.56 & 2.3 \\
\hline & $S$ & 81.0 & 2.08 & 0.29 & 2.93 & 0.83 & 2.1 \\
\hline \multirow{2}{*}{ IF (d) } & $\mathrm{R}$ & 1.1 & 0.30 & 0.09 & 3.15 & 0.26 & 158.5 \\
\hline & $S$ & 1.8 & 0.13 & $0.48^{* *}$ & 2.79 & 0.21 & 92.8 \\
\hline \multirow{2}{*}{$\mathrm{AP}(\mathrm{cm})$} & $\mathrm{R}$ & 181.6 & 16.33 & 6.54 & 99.64 & 0.37 & 5.5 \\
\hline & $S$ & 211.4 & 101.49 & $15.47 *$ & 84 & 0.84 & 4.3 \\
\hline \multirow{2}{*}{ SEN1 (1-10) } & $\mathrm{R}$ & 1.7 & 0.01 & 0.02 & 0.16 & 0.1 & 23.6 \\
\hline & $S$ & 2.7 & 0.01 & 0.01 & 0.23 & 0.19 & 17.9 \\
\hline \multirow{2}{*}{ SEN2 (1-10) } & $\mathrm{R}$ & - & - & - & - & - & - \\
\hline & $S$ & 3.9 & 0.00 & $0.11 * \star$ & 0.34 & 0.00 & 14.7 \\
\hline \multirow{2}{*}{ SEN3 (1-10) } & $\mathrm{R}$ & 3.2 & 0.05 & 0.001 & 0.37 & 0.37 & 19.2 \\
\hline & $S$ & 7.3 & 0.07 & $0.14 \star \star$ & 0.50 & 0.40 & 9.5 \\
\hline \multirow{2}{*}{ AUCSEN } & $\mathrm{R}$ & - & - & - & - & - & - \\
\hline & $S$ & 124.2 & 10.82 & $31.7 \star \star$ & 155.99 & 0.28 & 10.1 \\
\hline \multirow{2}{*}{ NDVI1 } & $\mathrm{R}$ & 0.31 & $4.3 \times 10^{-4}$ & $1.1 \times 10^{-3 * \star}$ & $2.0 \times 10^{-3}$ & 0.29 & 14.8 \\
\hline & $S$ & 0.24 & $1.8 \times 10^{-4}$ & $8.0 \times 10^{-4 * \star}$ & $7.2 \times 10^{-4}$ & 0.39 & 11.6 \\
\hline \multirow{2}{*}{ NDVI2 } & $\mathrm{R}$ & 0.46 & $2.0 \times 10^{-3}$ & $9.0 \times 10^{-6}$ & $3.6 \times 10^{-3}$ & 0.69 & 12.9 \\
\hline & $S$ & 0.41 & $1.8 \times 10^{-3}$ & $7.7 \times 10^{-4 * \star}$ & $2.7 \times 10^{-3}$ & 0.71 & 13.1 \\
\hline \multirow{2}{*}{ NDVI3 } & $\mathrm{R}$ & 0.53 & $2.8 \times 10^{-3}$ & $1.4 \times 10^{-18}$ & 0.01 & 0.69 & 13.6 \\
\hline & $S$ & 0.49 & $2.7 \times 10^{-3}$ & $2.1 \times 10^{-3 * *}$ & $4.1 \times 10^{-3}$ & 0.72 & 13.4 \\
\hline \multirow{2}{*}{ NDVI4 } & $\mathrm{R}$ & - & - & - & - & - & - \\
\hline & S & 0.59 & $2.8 \times 10^{-3}$ & $2.0 \times 10^{-3 * *}$ & $3.9 \times 10^{-3}$ & 0.74 & 10.8 \\
\hline \multirow{2}{*}{ AUCNDVI } & $\mathrm{R}$ & 12.15 & 1.47 & 0.00 & 1.95 & 0.75 & 11.5 \\
\hline & S & 12.00 & 1.22 & $1.03 * \star$ & 1.72 & 0.72 & 11.2 \\
\hline \multirow{2}{*}{ COMBO } & R & - & - & - & - & - & \\
\hline & $S$ & 19.71 & 2.90 & $1.77 \star \star$ & 8.96 & 0.65 & 15.4 \\
\hline
\end{tabular}

RG: rendimiento de grano, DA: días a antesis, IF: intervalo de floración, AP. altura de planta, SEN: senescencia, NDVI: índice de vegetación de diferencia normalizada, AUC (AUCSEN y AUCNDVI): área bajo la curva, $\sigma_{g}^{2}$ : varianza genética, $\sigma^{2}$ a e : varianza genotipo $\times$ ambiente, $\sigma_{e}^{2}:$ varianza residual, $H^{2}$ : heredabilidad en sentido amplio, CV: coeficiente de variación, *: significancia $\mathrm{P} \leq 0.05, * \star$ : significancia $\mathrm{P} \leq 0.01$. 
Cuadro 3. Correlación genética $\left(r_{g}\right)$ y fenotípica $\left(r_{f}\right)$ del rendimiento de grano con caracteres secundarios de líneas DH y cruzas de prueba (CP) bajo riego y sequía.

\begin{tabular}{|c|c|c|c|c|c|c|c|c|}
\hline \multirow[t]{2}{*}{ Carácter } & \multicolumn{2}{|c|}{ Líneas DH Riego } & \multicolumn{2}{|c|}{ CP Riego } & \multicolumn{2}{|c|}{ Líneas DH Sequía } & \multicolumn{2}{|c|}{ CP Sequía } \\
\hline & $r_{g}$ & $r_{f}$ & $r_{g}$ & $r_{f}$ & $r_{g}$ & $r_{f}$ & $r_{g}$ & $r_{f}$ \\
\hline DA & $0.78 * \star$ & -0.01 & -0.01 & -0.07 & $-0.19 *$ & $-0.17 \star$ & $-0.17 \star$ & -0.09 \\
\hline IF & 0 & 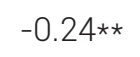 & $0.55 \star *$ & 0.15 & $-0.26 * *$ & $-0.17 \star$ & $0.73 * \star$ & 0.07 \\
\hline$A P$ & $0.30 * *$ & $0.26 * \star$ & -0.09 & 0.04 & $0.54 * *$ & $0.47 * *$ & $0.42 * \star$ & $0.34 * \star$ \\
\hline SEN1 & $-0.45 * \star$ & $-0.26 * \star$ & $0.99 * *$ & $0.42 \star \star$ & -0.10 & $-0.20 \star$ & $0.99 * *$ & $0.19 * *$ \\
\hline SEN2 & -0.12 & $-0.22 \star \star$ & $0.99 * *$ & $0.42 \star *$ & -0.11 & $-0.27 * *$ & 0.00 & -0.06 \\
\hline SEN3 & $-0.33 * *$ & $-0.33 \star \star$ & $0.59 * *$ & $0.27 \star \star$ & -0.12 & $-0.23 * *$ & $-0.26 * *$ & -0.12 \\
\hline AUCSEN & $-0.29 * *$ & $-0.33 \star \star$ & $0.99 * *$ & $0.46 * *$ & -0.14 & $-0.29 * *$ & $-0.58 * *$ & -0.06 \\
\hline NDVI1 & $0.77 \star \star$ & $0.59 * *$ & $0.99 * *$ & $0.67 * *$ & - & - & $0.99 * \star$ & $0.30 * *$ \\
\hline NDVI2 & $0.85 * *$ & $0.61 * *$ & $0.85 * *$ & $0.70 * *$ & - & - & $0.99 * \star$ & $0.44 * \star$ \\
\hline NDVI3 & $0.89 * *$ & $0.63^{* *}$ & $0.84 * *$ & $0.72 * *$ & - & - & $0.99 * *$ & $0.43^{* *}$ \\
\hline NDVI4 & $0.99 * *$ & $0.66 * *$ & - & - & - & - & $0.90 * \star$ & $0.42 * *$ \\
\hline AUCNDVI & $0.85 * \star$ & $0.65 * \star$ & $0.86 * *$ & $0.75 \star \star$ & - & - & $0.94 * \star$ & $0.44 * *$ \\
\hline COMBO & $0.68 * *$ & $0.60 * *$ & - & - & - & - & $0.96 * *$ & $0.55 * *$ \\
\hline
\end{tabular}

DA: días a antesis, IF: intervalo de floración, AP. altura de planta, SEN: senescencia, NDVI: índice de vegetación de diferencia normalizada, AUC (AUCSEN y AUCNDVI): área bajo la curva, *: significancia $\mathrm{P} \leq 0.05, * *$ : significancia $\mathrm{P} \leq 0.01$.

genotípica de 0.54 . El resto de las características tuvieron correlaciones bajas y negativas. Para las cruzas de prueba en riego, los caracteres IF, SEN1, NDVI (todas las tomas), AUCNDVI y COMBO presentaron correlaciones genéticas altas y positivas.

\section{Análisis y mapeo de QTLs asociados con tolerancia a sequía}

\section{QTLs para rendimiento}

En la población de líneas DH con riego se ubicó un QTL para rendimiento en el Bin 4.1 que explica el $17.8 \%$ de la variación fenotípica, el efecto de sustitución de esta región contribuye con un incremento de $0.12 \mathrm{t} \mathrm{ha}^{-1}$ aportado por LPS-C7-F64 (Cuadro 4). En la población de líneas DH bajo sequía se localizaron dos QTLs en los Bin 1.07 y 4.09 que participan con el 7.9 y $9.8 \%$ de la variación fenotípica con 0.2 y 0.6 t ha $^{-1}$, el primero proveniente de la línea CML495 y el segundo de LPS-C7-F64.

\section{QTL para días a antesis}

Se ubicó un QTL para días a antesis en las líneas $\mathrm{DH}$ bajo sequía, localizado en el Bin 1.03 (LOD 3.94) que explica el $12.9 \%$ de la variación fenotípica y que puede modificar los días a antesis en hasta un día (aditividad +0.88).

\section{QTLs para intervalo de floración}

En el intervalo de floración la línea CML495 contribuyó con dos QTLs favorables ubicados en los Bin 5.02/5.03 y 6.07/6.08 que explicaron 10.9 y $9.8 \%$ de la variación fenotípica, con efectos de asincronía de 0.48 y 0.44 d bajo condiciones de sequía.

\section{QTLs para senescencia}

En el Bin 4.5 se ubicó un QTL constitutivo de senescencia que explica el $13.8 \%$ de la variación fenotípica en AUCSEN_ LS y $10 \%$ en AUCSEN_LR, donde el alelo favorable proviene de la línea LPSC7F64. Otros QTLs importantes son los localizados en el Bin 9.02/9.03 provenientes de las líneas DH y cruzas de prueba (SEN1_LS, SEN3_LS y AUCSEN_ CPS) con una varianza fenotípica entre 7.8 y $13.2 \%$, donde los alelos favorables provienen de la línea CML495.

\section{QTLs para vigor}

Se localizaron siete QTLs para NDVI y ocho para altura de planta. Cinco QTLs de altura de planta y todos los de NDVI provienen de la línea CML495, lo que confiere un desarrollo vigoroso en la etapa vegetativa, que está altamente correlacionado con un incremento del rendimiento. En el Bin 1.03 se ubicaron los QTL de NDVI y altura de planta 
A)

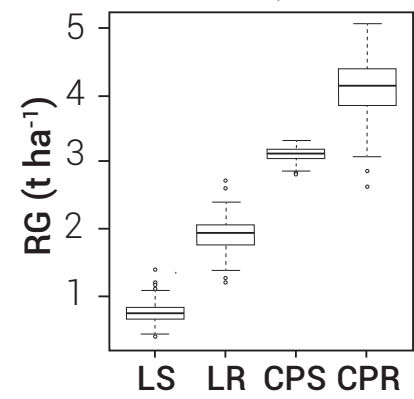

E)

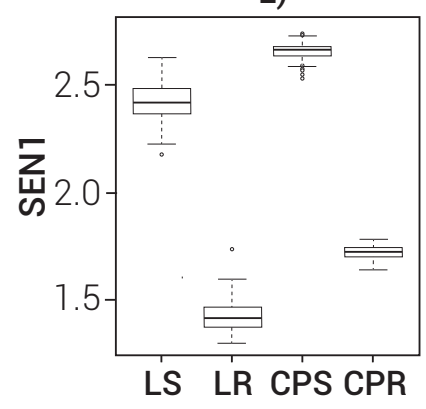

l)

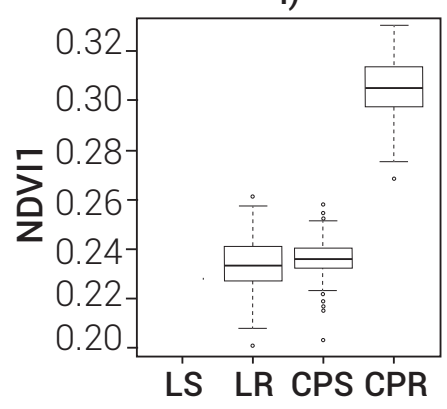

B)

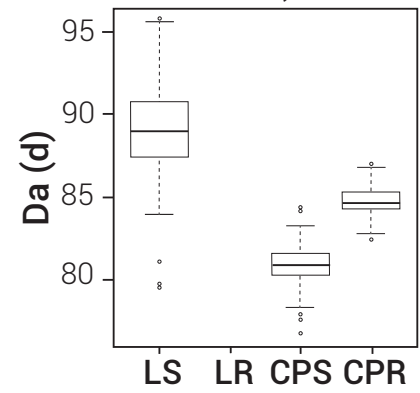

F)

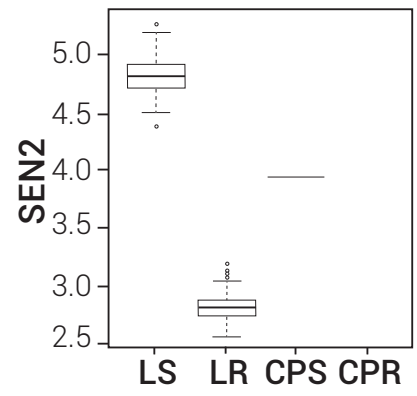

J)

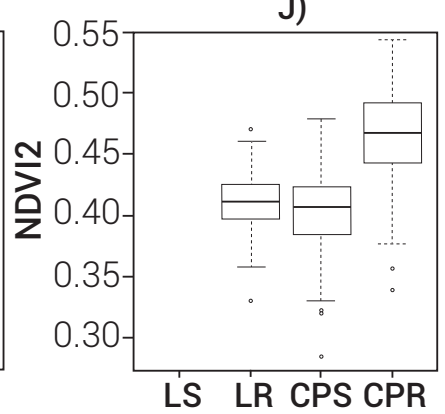

M)

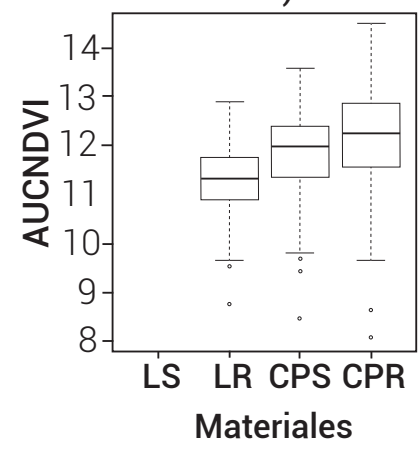

C)

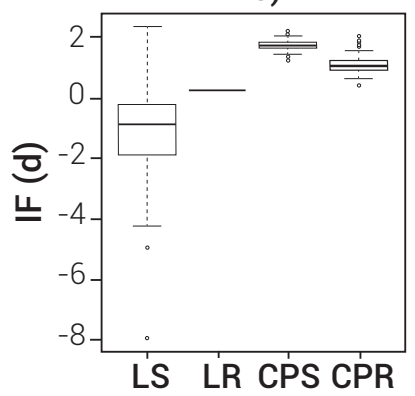

G)

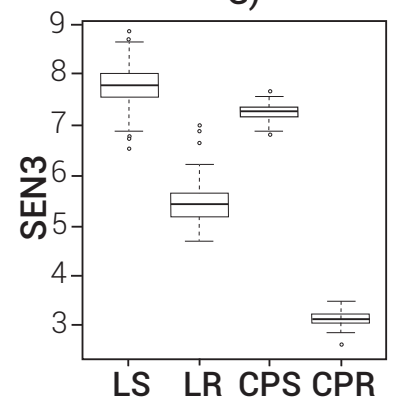

K)

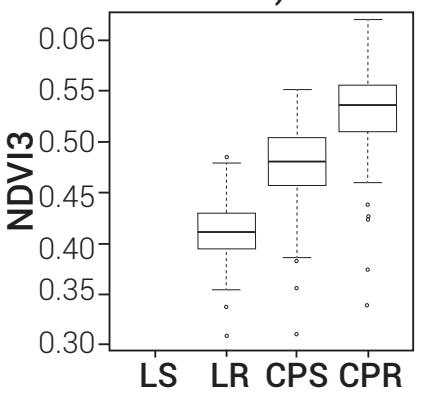

N)

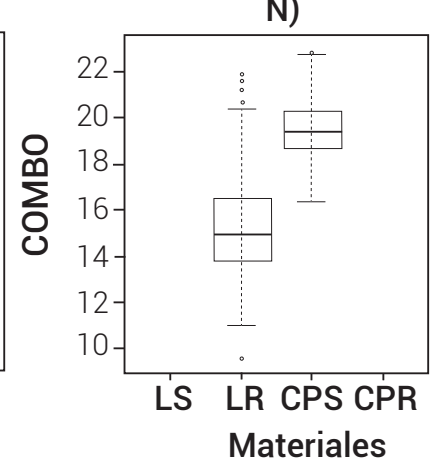

Figura 1. Diagramas de caja donde se muestran el primer cuartil, mediana, tercer cuartil y valores extremos de las mediciones de líneas DH y cruzas de prueba bajo sequía y riego. LS: líneas DH bajo sequía, LR: líneas DH bajo riego, CPS: cruzas de prueba bajo sequía, CPR: cruzas de prueba bajo riego. A) rendimiento de grano, B) días a antesis, C) intervalo de floración, D) altura de planta, E) senescencia SEN1, F) SEN2, G) SEN3, H) área bajo la curva AUCSEN, I) NDVI1, J) NDVI2, K) NDVI3, L) NDVI4, M) AUCNDVI, N) COMBO. 
Cuadro 4. QTLs obtenidos a través mapeo del intervalo compuesto (ICIM) de los rasgos de las líneas DH y cruzas de prueba bajo riego y sequía.

\begin{tabular}{|c|c|c|c|c|c|c|c|c|}
\hline Carácter & Cromosoma & $\mathrm{Bin}^{+}$ & $\begin{array}{l}\text { Posición } \\
(\mathrm{cM})^{++}\end{array}$ & $\begin{array}{l}\text { Intervalo del } \\
\text { marcador } \\
(\mathrm{cM})\end{array}$ & $\begin{array}{l}\text { Intervalo físico } \\
(\mathrm{Mb})^{\bullet}\end{array}$ & LOD“9 & $\mathrm{PVF}^{x}$ & $\operatorname{Add}^{\mathrm{x} x}$ \\
\hline SEN3_LR & 1 & $1.01 / 1.02$ & 131 & $128.25-131.25$ & $14.26-12.24$ & 3.11 & 8.66 & -0.11 \\
\hline Combo_CPS & 1 & $1.02 / 1.03$ & 118 & $116.51-118.28$ & $32.58-28.66$ & 2.57 & 7.49 & 0.28 \\
\hline DA_LS & 1 & 1.03 & 113 & $112.42-113.02$ & $38.49-36.77$ & 3.94 & 12.91 & 0.88 \\
\hline NDVI2_CPS & 1 & 1.03 & 107 & $104.12-107.04$ & $46.37-42.29$ & 3.48 & 9.99 & 0.01 \\
\hline AP_LR & 1 & 1.03 & 104 & $103.53-104.12$ & $49.77-46.37$ & 2.83 & 6.28 & 2.28 \\
\hline NDVI1_CPS & 1 & 1.03 & 104 & $103.53-104.12$ & $49.77-46.37$ & 2.72 & 7.2 & 0.002 \\
\hline NDVI3_CPS & 1 & 1.03 & 104 & $103.53-104.12$ & $49.77-46.37$ & 3.38 & 9.76 & 0.01 \\
\hline NDVI4_CPS & 1 & 1.03 & 104 & $103.53-104.12$ & $49.77-46.37$ & 3.37 & 8.89 & 0.01 \\
\hline AUCNDVI_CPS & 1 & 1.03 & 104 & $103.53-104.12$ & $49.77-46.37$ & 3.82 & 10.94 & 0.26 \\
\hline SEN1_LR & 1 & 1.06 & 75 & $73.88-75.65$ & $189.71-182.01$ & 2.83 & 8.78 & 0.02 \\
\hline RG_CPS & 1 & 1.07 & 55 & $53.86-55.06$ & $227.17-221.20$ & 2.72 & 7.92 & 0.02 \\
\hline AP_LR & 2 & 2.07 & 36 & $35.35-36.52$ & $204.30-201.75$ & 4.06 & 9.2 & -2.76 \\
\hline SEN3_CPS & 3 & 3.04 & 88 & $86.9-88.06$ & $95.83-49.74$ & 4.94 & 13.26 & 0.05 \\
\hline NDVI4_CPS & 3 & 3.04 & 87 & $86.9-88.06$ & $95.83-49.74$ & 2.53 & 6.62 & 0.01 \\
\hline SEN3_CPS & 3 & 3.07 & 52 & $51.95-53.11$ & $196.10-191.58$ & 2.96 & 7.71 & -0.04 \\
\hline AP_CPS & 3 & 3.09 & 21 & $19.93-22.3$ & $223.35-221.21$ & 2.83 & 7.63 & 1.56 \\
\hline$R G \_L R$ & 4 & 4.10 & 125 & $123.32-126.89$ & $239.21-239.97$ & 4.96 & 17.84 & -0.12 \\
\hline AUCSEN_LR & 4 & 4.05 & 43 & $42.97-43.54$ & $61.37-134.00$ & 3.2 & 9.86 & 1.62 \\
\hline SEN1_LS & 4 & 4.05 & 43 & $42.97-43.54$ & $61.37-134.00$ & 3.4 & 10.01 & 0.03 \\
\hline SEN3_LS & 4 & 4.05 & 43 & $42.97-43.54$ & $61.37-134.00$ & 3.87 & 12 & 0.13 \\
\hline AUCSEN_LS & 4 & 4.05 & 43 & $42.97-43.54$ & $61.37-134.00$ & 4.47 & 13.81 & 2.27 \\
\hline Combo_LR & 4 & 4.05 & 45 & $43.54-46.44$ & $110.49-148.67$ & 5 & 12.99 & -0.72 \\
\hline AP_LR & 4 & $4.05 / 4.06$ & 47 & $46.44-47.02$ & $148.67-155.30$ & 5.13 & 11.64 & -3.1 \\
\hline SEN1_LR & 4 & 4.06 & 49 & $47.02-49.38$ & $155.30-156.72$ & 3.23 & 9.98 & 0.02 \\
\hline AP_LS & 4 & 4.06 & 74 & $66.57-4.25$ & $170.14-167.07$ & 4.19 & 12.57 & -2.52 \\
\hline IF_CPS & 4 & 4.08 & 95 & $92.88-95.39$ & $180.43-181.36$ & 2.53 & 6.78 & 0.04 \\
\hline RG_LS & 4 & 4.09 & 108 & $107.29-108.46$ & $209.56-222.18$ & 2.95 & 9.79 & -0.06 \\
\hline RG_LS & 4 & 4.09 & 115 & $112.11-115.04$ & $233.93-235.20$ & 2.62 & 8.75 & -0.04 \\
\hline SEN1_CPS & 5 & 5.01 & 84 & $84-87.53$ & $5.29-4.54$ & 2.51 & 7.78 & -0.01 \\
\hline AUCSEN_LR & 5 & 5.03 & 62 & $61.63-62.88$ & $69.09-62.76$ & 2.68 & 8.07 & 1.47 \\
\hline NDVI1_CPS & 5 & 5.03 & 60 & $59.89-61.63$ & $77.67-69.09$ & 3.43 & 9.21 & 0.002 \\
\hline IF_LS & 5 & $5.03 / 5.04$ & 59 & $58.14-59.89$ & $89.17-77.67$ & 3.32 & 10.91 & 0.48 \\
\hline SEN3_LR & 5 & 5.04 & 50 & $49.92-51.69$ & $168.23-165.61$ & 2.98 & 8.22 & 0.11 \\
\hline Combo_LR & 5 & 5.05 & 42 & $41.57-43.98$ & $174.86-172.70$ & 4.56 & 11.61 & -0.68 \\
\hline AP_LS & 6 & 6.01 & 61 & $60.72-63.61$ & $60.18-30.46$ & 3.76 & 11.12 & 2.35 \\
\hline SEN3_LR & 6 & 6.04 & 44 & $43.44-44.03$ & $110.16-106.67$ & 2.5 & 6.85 & 0.09 \\
\hline IF_LS & 6 & $6.07 / 6.08$ & 1 & $0-1.18$ & $167.57-166.33$ & 3.24 & 9.77 & 0.44 \\
\hline
\end{tabular}


Cuadro 4. Continúa.

\begin{tabular}{|c|c|c|c|c|c|c|c|c|}
\hline Carácter & Cromosoma & $\operatorname{Bin}^{+}$ & $\begin{array}{l}\text { Posición } \\
(\mathrm{cM})^{++}\end{array}$ & $\begin{array}{l}\text { Intervalo del } \\
\text { marcador } \\
\text { (cM) }\end{array}$ & $\begin{array}{l}\text { Intervalo físico } \\
(\mathrm{Mb})^{9}\end{array}$ & LOD“9 & $P V F^{x}$ & Add $d^{x}$ \\
\hline AP_LR & 7 & 7.03 & 49 & $48.07-49.26$ & $136.38-137.35$ & 5.87 & 13.59 & 3.34 \\
\hline Combo_LR & 7 & 7.03 & 49 & $48.07-49.26$ & $136.38-137.35$ & 3.94 & 9.89 & 0.62 \\
\hline AP_CPS & 8 & $8.04 / 8.05$ & 73 & $72.61-74.46$ & $116.33-123.33$ & 2.73 & 7.35 & 1.53 \\
\hline AUCSEN_CPS & 9 & $9.02 / 9.03$ & 29 & $29-30.75$ & $23.11-32.95$ & 2.70 & 7.83 & -0.35 \\
\hline IF_CPS & 9 & 9.06 & 62 & $61.79-62.98$ & $146.48-147.24$ & 2.95 & 8.04 & -0.04 \\
\hline
\end{tabular}

LR: líneas DH bajo riego, LS: líneas bajo sequía, CPR: cruzas de prueba bajo rieg,; CPS: cruzas de prueba bajo sequía, RG: rendimiento de grano, DA: días a antesis, IF: intervalo de floración, AP. altura de planta, SEN: senescencia, AUC (AUCSEN y AUCNDVI) área bajo la curva, NDVI: índice de

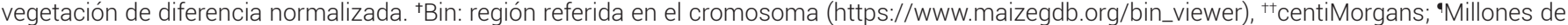
pares de bases, "valor LOD, ×Porción de la varianza fenotípica del QTL, ${ }^{x \times E f e c t o ~ a d i t i v o ~ d e ~ c a d a ~ Q T L . ~}$

que explican hasta el $10.9 \%$ (AUCNDVI_CPS) y $6.3 \%$ (AP_ LR) de la variación fenotípica y confiere un incremento en altura de planta de $2.28 \mathrm{~cm}$. Tal alelo podría implicar un QTL constitutivo proveniente de la línea CML495. La región 5.03 de NDVI1_CPS explica el $9.2 \%$ de la variación. Estas dos importantes regiones también fueron localizadas en el trabajo de Trachsel et al. (2016).

\section{QTLs para altura de planta}

Para condiciones de sequía se ubicaron en los Bin 3.09 4.06, 6.01 y 8.04/8.05, donde la variación fenotípica explicada fue de 7.4 y $12.6 \%$ y valores aditivos de 1.5 y $2.5 \mathrm{~cm}$. Otro QTL importante aportado por la línea CML495 es de AP_LR en el Bin 7.03 que explica el $13.6 \%$ de la variación fenotípica y un incremento de $3.34 \mathrm{~cm}$. Esta región también fue localizada por Almeida et al. (2013) para altura de planta.

\section{QTLs para COMBO}

Los QTLs que integran la relación vigor/senescencia para riego se encontraron junto con las de altura de planta en los Bin 4.05/4.06 y 7.03 y de manera individual en el 5.05, que juntos explicaron el $34.5 \%$ de la variación fenotípica. Para sequía se ubicó un QTL en el Bin 1.02/1.03, que proporciona el $7.5 \%$ de la variación fenotípica.

La heredabilidad del rendimiento se considera moderada para las líneas DH bajo condiciones de riego y sequía, en tanto que en las CP bajo riego la heredabilidad resultante es considerada como alta, y para sequía se comportó como baja, lo que pudo deberse a las altas varianzas residual y de interacción $\mathrm{G} \times$ A, como lo reportan Lafitte et al. (2003). Las heredabilidades de los caracteres secundarios en las cruzas de prueba en su mayoría fueron altas bajo sequía y altamente correlacionadas con el rendimiento. En esta investigación se pusieron de manifiesto correlaciones fenotípicas y genotípicas importantes entre el rendimiento, el intervalo de floración (líneas DH), altura de planta, SEN3, AUCSEN (CP), NDVI2, NDVI3, NDVI4, AUCNDVI y COMBO, los que también se muestran en otros trabajos (Almeida et al., 2013; Lu et al., 2011; Messmer et al., 2011; Trachsel et al., 2016).

En el presente estudio los caracteres secundarios NDVI y altura de planta fueron los que mejor correlación tuvieron con el rendimiento y con alta heredabilidad, tanto en estrés hídrico como en condiciones óptimas de humedad; la misma tendencia se presentó en la relación vigor y senescencia, referida como COMBO. El contenido de clorofila y la senescencia retrasada están correlacionados con NDVI y altura de planta, y éstos a su vez con el rendimiento, como lo describe Lu et al. (2011), por lo que, utilizar a COMBO como carácter secundario ofrece una mejor identificación de genotipos tolerantes a sequía. De los QTLs de rendimiento y caracteres secundarios de rendimiento con alto valor LOD y porcentaje de variación fenotípica se ubicaron varios segmentos contenidos en los Bin 1.02/1.03, 1.07, 4.05, 4.10 y 7.03 .

\section{QTL rendimiento bajo sequía Bin 1.07}

Este QTL para rendimiento se ha identificado en otros estudios bajo condiciones normales (Frascaroli et al., 2007) y en condiciones de estrés (Zaidi et al., 2015). En la región del cromosoma 1 se encuentra el gen agps/1, que está relacionado con el incremento de almidón en el grano (Huang et al., 2014; Liu et al., 2016). 


\section{QTL rendimiento bajo riego Bin 4.10.}

Este QTL es un candidato para ser introducido en líneas elite de maíz. Almeida et al. (2013) lo ubicaron en la línea CML504 en la posición 239.24 - 242.02 Mb. En esta región se ubica el gen NRT2.1. El estudio de Sorgonà et al. (2011) sugiere que NRT2.1 es un componente importante del sistema transportador de alta afinidad (iHATS) para la absorción de $\mathrm{NO}_{3}-$ a lo largo del eje de la raíz del maíz.

\section{QTL Bin 1.03 altura de planta, NDVI (vigor) y COMBO_CPS}

Esta región está relacionada con el rendimiento en condiciones de sequía (Millet et al., 2016; Zhu et al., 2011) y en condiciones normales (Semagn et al., 2013) y también se han ubicado QTLs para altura de planta (Frascaroli et al., 2007), stay Green (Almeida et al., 2014) y senescencia (Trachsel et al., 2016). Los efectos de esta región indican una favorable capacidad de crecimiento y producción bajo condiciones de sequía.

\section{QTL 4.05 COMBO y senescencia}

En el Bin 4.05 se han ubicado varios QTLs asociados a rendimiento bajo sequia (Zhu et al., 2011), rendimiento en condiciones normales (Chen et al., 2016), altura de planta (Lu et al., 2012) y senescencia (Trachsel et al., 2016). En esta región se ubica el gen nnr 7 que está relacionado con la absorción de nitrógeno en maíz (Liseron-Monfils et al., 2013).

\section{QTL 7.03 COMBO}

Se ha localizado un QTL para altura de planta tanto en riego como en sequía (Almeida et al., 2014; Peiffer et al., 2014), rendimiento en sequía (Almeida et al., 2013) en el que se encuentra el gen glutation transferasa 23 (gst23) (Chen et al., 2012; Varga et al., 2012) y fitoeno sintasa 3 (psy3), donde se demostró que la acumulación de ABA en raíz está limitada por la expresión de PSY3 en condiciones de estrés por sequía (Li et al., 2009).

\section{CONCLUSIONES}

Los caracteres secundarios NDVI, altura de planta, senescencia (Sen3 y AUCSEN) y COMBO proporcionaron mejor información y permitieron identificar genotipos con buen rendimiento de líneas DH y cruzas de prueba en etapas tempranas bajo condiciones de sequía y régimen de humedad adecuado. Los QTLs ubicados en los cromosomas 1, 4, y 7 son los de mayor relevancia y están relacionados con vigor y rendimiento; éstos pueden ser útiles en la introgresión asistida por marcadores en líneas e híbridos de trópico para aumentar el rendimiento bajo condiciones de sequía y de buena humedad. Los resultados de rendimiento y los caracteres secundarios son menos sesgados al utilizar las mediciones de líneas y cruzas de prueba, lo que reduce la interacción con alelos deletéreos.

\section{BIBLIOGRAFÍA}

Almeida G. D., D. Makumbi, C. Magorokosho, S. Nair, A. Borém, J. M. Ribaut, M. Bänziger, B. M. Prasanna, J. Crossa and R. Babu (2013) QTL mapping in three tropical maize populations reveals a set of constitutive and adaptive genomic regions for drought tolerance. Theoretical and Applied Genetics 126:583-600 https://doi.org/10.1007/s00122-012-2003-7

Almeida G. D., S. Nair, A. Borém, J. Cairns, S. Trachsel, J. M. Ribaut, M. Bänziger, B. M. Prasanna, J. Crossa and R. Babu (2014) Molecular mapping across three populations reveals a QTL hotspot region on chromosome 3 for secondary traits associated with drought tolerance in tropical maize. Molecular Breeding 34:701-715 https://doi.org/10.1007/s11032-014-0068-5

Alvarado G., M. López, M. Vargas, A. Pacheco, F. Rodríguez, J. Burgueño and J. Crossa (2015) META-R (Multi Environment Trial Analysis whit $\mathrm{R}$ for Windows.) Version 5.0. CIMMYT Research Sotware. International Maize and Wheat Improvement Center. El Batán Texcoco, México. http://hdl.handle.net/11529/10201 (January 2020).

Awika J. M. (2011) Major cereal grains production and use around the world. In: Advances in Cereal Science: Implications to Food Processing and Health Promotion. American Chemical Society. Washington, D. C. pp:1-13, https://doi.org/doi:10.1021/bk2011-1089.ch001

Bänziger M., G. O. Edmeades, D. Beck y M. Bellon. (2012) Mejoramiento para Aumentar la Tolerancia a Sequía y a Deficiencia de Nitrógeno en el Maíz. De la Teoria a la Práctica. Centro Internacional de Mejoramiento de Maíz y Trigo. México, D. F. 68 p.

Chen J. H., H. W. Jiang, E. J. Hsieh, H. Y. Chen, C. T. Chien, H. L. Hsieh and T. P. Lin (2012) Drought and salt stress tolerance of an Arabidopsis glutathioneS-transferase U17 knockout mutant are attributed to the combined effect of glutathione and abscisic acid. Plant Physiology 158:340-351, https://doi.org/10.1104/pp.111.181875

Chen L., Y. Li, C. Li, X. Wu, W. Qin, X. Li, ... and T. Wang (2016) Fine-mapping of qGW4.05, a major QTL for kernel weight and size in maize. BMC Plant Biology 16:81, https://doi.org/10.1186/s12870-016-0768-6

CIMMYT, Centro Internacional de Mejoramiento de Maíz y Trigo (2005) Laboratory Protocols: CIMMYT Applied Molecular Genetics Laboratory. 3rd edition. CIMMYT. México, D. F. 102 p.

Daryanto S., L. Wang and P. A. Jacinthe (2016) Global synthesis of drought effects on maize and wheat production. PLOS ONE 11 (5):e0156362, https://doi.org/10.1371/journal.pone.0156362

Duvick D. N. (2005) Genetic progress in yield of United States maize (Zea mays L.). Maydica 50:193-202.

Elshire R. J., J. C. Glaubitz, Q. Sun, J. A. Poland, K. Kawamoto, E. S. Buckler and S. E. Mitchell (2011) A robust, simple genotyping-by-sequencing (GBS) approach for high diversity species. PLOS ONE 6(5):e19379, https://doi.org/10.1371/journal.pone.0019379

FAOSTAT, Food and Agriculture Organization Corporate Statistical Database (2015) Crops. Food and Agriculture Organization of the United Nations. Rome. http://www.fao.org/faostat/en/\#data/QC/ visualize. (October 2016).

Frascaroli E., M. A. Canè, P. Landi, G. Pea, L. Gianfranceschi, M. Villa, M. Morgante and M. E. Pè (2007) Classical genetic and quantitative trait loci analyses of heterosis in a maize hybrid between two elite inbred lines. Genetics 176:625 644, https://doi.org/10.1534/genetics.106.064493

Frascaroli E., M. A. Canè, M. E. Pè, G. Pea, M. Morgante and P. Landi (2009) QTL detection in maize testcross progenies as affected by related and unrelated testers. Theoretical and Applied Genetics 118:993-1004, https://doi.org/10.1007/s00122-008-0956-3

Glaubitz J. C., T. M. Casstevens, F. Lu, J. Harriman, R. J. Elshire, Q. Sun and 
E. S. Buckler (2014) TASSEL-GBS: a high capacity genotyping by sequencing analysis pipeline. PLOS ONE 9(2):e90346, https://doi.org/10.1371/journal.pone.0090346

Huang B., T. A. Hennen-Bierwagen and A. M. Myers (2014) Functions of multiple genes encoding ADP-glucose pyrophosphorylase subunits in maize endosperm, embryo, and leaf. Plant Physiology 164:596-611, https://doi.org/10.1104/pp.113.231605

Inzunza-Ibarra M. A., Ma. M. Villa-Castorena, E. A. Catalán-Valencia, R. LópezLópez y E. Sifuentes-Ibarra (2018) Rendimiento de grano de maíz en deficit hídrico en el suelo en dos etapas de crecimiento. Revista Fitotecnia Mexicana 41:283-290.

IPCC, Internationtal Panel on Climate Change (2012) Managing the Risks of Extreme Events and Disasters to Advance Climate Change Adaptation. Special Report of the Intergovernmental Panel on Climate Change. C. B. Field, V. Barros, T. F. Stocker and Q. Dahe (eds.). Cambridge University Press. Cambridge, UK. 582 p, https://doi.org/10.1017/CB09781139177245

Kosambi D. D. (1943) The estimation of map distance from recombination values. Annals of Eugenics 12:172-175, https://doi.org/10.1111/j.1469-1809.1943.tb02321.x

Lafitte R., A. Blum and G. Atlin (2003) Using secondary traits to help identify drought-tolerant genotypes. In: Breeding Rice for Drought-Prone Environments. K. S. Fischer, R. Lafitte, S. Fukai, G. Atlin and B. Hardy (eds.). International Rice Research Institute. Los Baños, Philippines. pp:37-48.

Li F., O. Tsfadia and E. T. Wurtzel (2009) The phytoene synthase gene family in the grasses: subfunctionalization provides tissuespecific control of carotenogenesis. Plant Signaling and Behavior 4:208-211, https://doi.org/10.4161/psb.4.3.7798

Li H., G. Ye and J. Wang (2007) A modified algorithm for the improvement of composite interval mapping. Genetics 175:361-374, https://doi.org/10.1534/genetics.106.066811

Liseron-Monfils C., Y. M. Bi, G. S. Downs, W. Wu, T. Signorelli, G. Lu, ... M. N. Raizada (2013) Nitrogen transporter and assimilation genes exhibit developmental stage-selective expression in maize (Zea mays L.) associated with distinct cis-acting promoter motifs. Plant Signaling and Behavior 8:10, https://doi.org/10.4161/psb.26056

Liu N., Y. Xue, Z. Guo, W. Li and J. Tang (2016) Genome-wide association study identifies candidate genes for starch content regulation in maize kernels. Frontiers in Plant Science 7:1046, https://doi. org/10.3389/fpls.2016.01046

Lu Y., Z. Hao, C. Xie, J. Crossa, J. L. Araus, S. Gao, ... Y. Xu (2011) Largescale screening for maize drought resistance using multiple selection criteria evaluated under water-stressed and wellwatered environments. Field Crops Research 124:37-45, https://doi.org/10.1016/j.fcr.2011.06.003

Lu Y., J. Xu, Z. Yuan, Z. Hao, C. Xie, X. Li, T. Shah, H. Lan, S. Zhang, T. Rong and Y. Xu (2012) Comparative LD mapping using single SNPs and haplotypes identifies QTL for plant height and biomass as secondary traits of drought tolerance in maize. Molecular Breeding 30:407-418, https://doi.org/10.1007/s11032-011-9631-5

Messmer R., Y. Fracheboud, M. Bänziger, P. Stamp and J. M. Ribaut (2011) Drought stress and tropical maize: QTLs for leaf greenness, plant senescence, and root capacitance. Field Crops Research 124:93-103, https://doi.org/10.1016/j.fcr.2011.06.010

Millet E. J., C. Welcker, W. Kruijer, S. Negro, A. Coupel-Ledru, S. D. Nicolas, ... and F. Tardieu (2016) Genome-wide analysis of yield in Europe: allelic effects vary with drought and heat scenarios. Plant Physiology 172:749-764, https://doi.org/10.1104/pp.16.00621
Olivera V. S. M. (2013) La Productividad del Maíz de Temporal en México: Repercusiones del Cambio Climático. División de Desarrollo Sostenible y Asentamientos Humanos, CEPAL. Santiago de Chile. 24 p.

Peiffer J. A., M. C. Romay, M. A. Gore, S. A. Flint-Garcia, Z. Zhang, M. J. Millard, C. A. C. Gardner, M. D. McMullen, J. B. Holland, P. J. Bradbury and E. S. Buckler (2014) The genetic architecture of maize height. Genetics 196:1337-1356, https://doi.org/10.1534/genetics.113.159152

Ramírez-Díaz J. L., V. A. Vidal-Martínez, I. Alemán-de-la-Torre, A. LedesmaMiramontes, N. O. Gómez-Montiel, Y. Salinas-Moreno, ... y A. Ruiz-Corral (2019) Selección de líneas y cruzas de maízcombinando las pruebas de mestizos y cruzas dialélicas. Revista Fitotecnia Mexicana 42:335-346, https://doi.org/10.35196/rfm.2019.4.335-346

Reyes A., C. D. Messina, G. L. Hammer, L. Liu, E. van Oosterom, R. Lafitte and M. Cooper (2015) Soil water capture trends over 50 years of single-cross maize (Zea mays L.) breeding in the US corn-belt. Journal of Experimental Botany 66:7339-7346, https://doi.org/10.1093/jxb/erv430

Ribaut J. M. and M. Ragot (2007) Marker-assisted selection to improve drought adaptation in maize: the backcross approach perspectives, limitations, and alternatives. Journal of Experimental Botany 58:351-360, https://doi.org/10.1093/jxb/erl214

Semagn K., Y. Beyene, M. L. Warburton, A. Tarekegne, S. Mugo, B. Meisel, P. Sehabiague and B. M. Prasanna (2013) Metaanalyses of QTL for grain yield and anthesis silking interval in 18 maize populations evaluated under waterstressed and well-watered environments. BMC Genomics 14:313, https://doi.org/10.1186/1471-2164-14-313

Sorgonà A., A. Lupini, F. Mercati, L. Di Dio, F. Sunseri and M. R. Abenavoli (2011) Nitrate uptake along the maize primary root: an integrated physiological and molecular approach. Plant, Cell and Environment 34:1127-1140, https://doi.org/10.1111/j.1365-3040.2011.02311.x

Trachsel S., D. Sun, F. M. SanVicente, H. Zheng, G. N. Atlin, E. A. Suarez, R. Babu and X. Zhang (2016) Identification of QTL for early vigor and stay-green conferring tolerance to drought in two connected advanced backcross populations in tropical maize (Zea mays L.). PLoS ONE 11(3):e0149636, https://doi.org/10.1371/journal.pone.0163400

Varga B., T. Janda, E. László and O. Veisz (2012) Influence of abiotic stresses on the antioxidant enzyme activity of cereals. Acta Physiologiae Plantarum 34:849-858, https://doi.org/10.1007/s11738-011-0882-x

Zaidi P. H., Z. Rashid, M. T. Vinayan, G. D. Almeida, R. K. Phagna and R. Babu (2015) QTL mapping of agronomic waterlogging tolerance using recombinant inbred lines derived from tropical maize (Zea mays L.) germplasm. PLoS ONE 10(4):e0124350, https://doi.org/10.1371/journal.pone.0124350

Zeng Z. B., C. H. Kao and C. J. Basten (1999) Estimating the genetic architecture of quantitative traits. Genetics Research 74:279289, https://doi.org/10.1017/s0016672399004255

Zhang X., P. Pérez-Rodríguez, K. Semagn, Y. Beyene, R. Babu, M. A. LópezCruz, ... and J. Crossa (2015) Genomic prediction in biparental tropical maize populations in water-stressed and well-watered environments using low-density and GBS SNPS. Heredity 114:291-299, https://doi.org/10.1038/hdy.2014.99

Zhu J. J., X. P. Wang, C. X. Sun, X. M. Zhu, M. Li, G. D. Zhang, ... and Z. L. Wang (2011) Mapping of QTL associated with drought tolerance in a semi-automobile rain shelter in maize (Zea mays L.). Agricultural Sciences in China 10:987-996, https://doi.org/10.1016/S1671-2927(11)60085-0 